\title{
Article
}

\section{Degree affinity number of certain 2-regular graphs}

\author{
Johan Kok \\ Independent Mathematics Researcher, City of Tshwane, South Africa \& Visiting Faculty at CHRIST (Deemed to be a \\ University), Bangalore, India.; johan.kok@christuniversity.in; Tel.: +27646547285
}

Received: 15 October 2020; Accepted: 20 November 2020; Published: 14 December 2020.

\begin{abstract}
This paper furthers the study on a new graph parameter called the degree affinity number. The degree affinity number of a graph $G$ is obtained by iteratively constructing graphs, $G_{1}, G_{2}, \ldots, G_{k}$ of increased size by adding a maximal number of edges between distinct pairs of distinct vertices of equal degree. Preliminary results for certain 2-regular graphs are presented.
\end{abstract}

Keywords: Degree affinity edge, degree affinity number.

MSC: 05C15, 05C38, 05C75, 05C85.

\section{Introduction}

I $\mathrm{t}$ is assumed that the reader is familiar with the general notation and concepts in graphs. Good references are [1-3]. Throughout the study only finite, simple and undirected graphs will be considered. A paper which introduces the notion of the degree affinity number of a graph has been communicated, (see [4]).

It is known that a graph of order $n \geq 2$ has at least two vertices of equal degree. We recall that if two non-adjacent vertices $u, v \in V(G)$ with $\operatorname{deg}_{G}(u)=\operatorname{deg}_{G}(v)$ exist, then the added edge $u v$ to obtain $G^{\prime}$ is called a degree affinity edge. For ease of reference we also recall the maximal degree affinity convention.

\section{Maximal degree affinity convention (MDAC)}

For a graph $G$ the $1^{\text {st }}$-iteration is the addition of degree affinity edges in respect of a maximal number of absolute distinct pairs of distinct non-adjacent vertices of equal degree, if such exist [4]. The graph obtained is labeled $G_{1}$. Hence, by the same convention it is possible to construct $G_{i}$ from $G_{i-1}$ provided that at least one (absolute distinct) pair of distinct non-adjacent vertices of equal degree exists in $G_{i-1}$. The MDAC terminates on the $k^{\text {th }}$-iteration if no further edges can be added.

We recall certain important results from [4].

Theorem 1. [4] For an even cycle $C_{n}, n \geq 4$ the MDAC exhausts after $k=n-3$ iterations, $\eta\left(C_{n}\right)_{n, \text { even }}=\frac{n(n-3)}{2}$ and $G_{n-3} \cong K_{n}$.

Corollary 1. [4] For an odd cycle $C_{n}, n \geq 5$ the MDAC exhausts after $k=n-3$ iterations and $\eta\left(C_{n}\right)_{n, o d d}=$ $\frac{(n-2)(n-3)}{2}$.

If a graph $G$ has structural complexity, then finding $\eta(G)$ could be simplied by considering $\bar{G}$. However the dual problem must be considered. The dual to finding $\eta(G)$ is the deletion of the maximum number degree affinity edges from $\bar{G}$. The procedure is the iterative inverse of the MDAC and is denoted by, MDAC ${ }^{-1}$. If a null graph (edgeless graph) results we say $\bar{G}$ reached nullness.

Theorem 2. A graph $G$ reaches completeness on exhaustion of the $M D A C$ if and only if $\bar{G}$ reaches nullness on exhaustion of the $M D A C^{-1}$.

Proof. If $G$ reaches completeness on exhaustion of the MDAC then the set of degree affinity edges added is exactly, $E(\bar{G})$. By listing the degree affinity edges say $s_{i}$ added to $G$ per MDAC iteration $i=1,2,3, \ldots, k$ the inverse iterative deletion of degree affinity edge-lists $s_{j}$ in $\bar{G}$ for $j=k, k-1, k-2, \ldots, 1$, results in nullness. 
The converse follows through similar reasoning. Therefore the result.

In this paper we further the study in [4] for certain 2-regular graphs.

\section{On regular graphs}

To study the disjoint union of graphs, we distinguish between degree affinity edges internal to a graph $G$ and those external to $G$. Let $V(G)=\left\{v_{i}: 1 \leq i \leq n\right\}$ and $V(H)=\left\{u_{j}: 1 \leq j \leq m\right\}$. In the disconnected graph $G \cup H$ and through all iterations of the MDAC applied thereto, degree affinity edges of the form $v_{i} v_{k}$ or $u_{j} u_{t}$ are called internal to $G$ or $H$, respectively. Degree affinity edges of the form $v_{i} u_{j}$ are called external to both $G$ and $H$. Furthermore, if all vertex degrees $\operatorname{deg}_{G}\left(v_{i}\right), v_{i} \in V(G)$ are weighted by a constant $a \in \mathbb{N}$ we denote the graph with weighted degrees by, $G^{+a}$.

Lemma 1. If graph $G$ of order $n$ has degree sequence $\left(\operatorname{deg}_{G}\left(v_{i}\right): \operatorname{deg}_{G}\left(v_{i}\right) \geq \operatorname{deg}_{G}\left(v_{i+1}\right), 1 \leq i \leq n-1\right)$ and $G^{+a}$ has degree sequence $\left(\operatorname{deg}_{G}\left(v_{i}\right)+a: \operatorname{deg}_{G}\left(v_{i}\right) \geq \operatorname{deg}_{G}\left(v_{i+1}\right), 1 \leq i \leq n-1\right)$ then the degree affinity properties of $G^{+a}$ are identical to that of $G$.

Proof. Since

(a) $v_{i} v_{j} \in E(G)$ and $\operatorname{deg}_{g}\left(v_{i}\right)=\operatorname{deg}_{G}\left(v_{j}\right)$ in $G \Leftrightarrow v_{i} v_{j} \in E\left(G^{+a}\right)$ and $\operatorname{deg}_{g}\left(v_{i}\right)+a=\operatorname{deg}_{G}\left(v_{j}\right)+a$ in $G^{+a}$ or;

(b) $v_{i} v_{j} \in E(G)$ and $\operatorname{deg}_{g}\left(v_{i}\right) \neq \operatorname{deg}_{G}\left(v_{j}\right)$ in $G \Leftrightarrow v_{i} v_{j} \in E\left(G^{+a}\right)$ and $\operatorname{deg}_{g}\left(v_{i}\right)+a \neq \operatorname{deg}_{G}\left(v_{j}\right)+a$ in $G^{+a}$ or;

(c) $v_{i} v_{j} \notin E(G)$ and $\operatorname{deg}_{g}\left(v_{i}\right)=\operatorname{deg}_{G}\left(v_{j}\right)$ in $G \Leftrightarrow v_{i} v_{j} \notin E\left(G^{+a}\right)$ and $d e g_{g}\left(v_{i}\right)+a=\operatorname{deg}_{G}\left(v_{j}\right)+a$ in $G^{+a}$ or;

(d) $v_{i} v_{j} \notin E(G)$ and $\operatorname{deg}_{g}\left(v_{i}\right) \neq \operatorname{deg}_{G}\left(v_{j}\right)$ in $G \Leftrightarrow v_{i} v_{j} \notin E\left(G^{+a}\right)$ and $\operatorname{deg}_{g}\left(v_{i}\right)+a \neq \operatorname{deg}_{G}\left(v_{j}\right)+a$ in $G^{+a}$,

the result follows immediately.

An immediate consequence of Lemma 1 follows.

Theorem 3. Let graphs $G$ and $H$ both be of order $n$ and r-regular then, $\eta(G \cup H)=\eta(G)+\eta(H)+n^{2}$.

Proof. Clearly, the disjoint union $G \cup H$ is of order $2 n$. Hence, for each of the initial $n$ iterations there exist $n$ distinct pairs of distinct vertices $\{u, v\}, u \in V(G), v \in V(H)$ such that $u v \notin E(G \cup H)$ and $\operatorname{deg}_{(G \cup H)_{i-1}}(u)=$ $\operatorname{deg}_{(G \cup H)_{i-1}}(v)=\operatorname{deg}_{G}(u)+(i-1)=\operatorname{deg}_{H}(v)+(i-1)$. Therefore, after the initial $n$ iterations all the possible degree affinity edges between $G$ and $H$ have been added. Without loss of generality, the graph $G$ can be viewed as a graph with weighted vertex degrees i.e., $G^{+n}$. By Lemma 1 the degree affinity properties of $G^{+n}$ are identical to that of $G$, (similarly for $H^{+n}$ ). The MDAC is now simultaneously applied to $G^{+n}$ and $H^{+n}$. Thus, $\eta(G \cup H)=\eta(G)+\eta(H)+n^{2}$.

Applying Theorem 3 could present difficulty. It is easy to see that if both graphs $G$ and $H$, independently reach completeness on exhaustion of the MDAC, the grouped iterations i.e., (a) first apply MDAC independently to $G$ and $H$, (b) thereafter apply MDAC between $\mathbb{A}(G)$ and $\mathbb{A}(H)$ or interchanging the grouped iterations, yield the same result. This observations does not hold in general. Consider the two 3-regular graphs of order 6 (Figures 1 and 2).

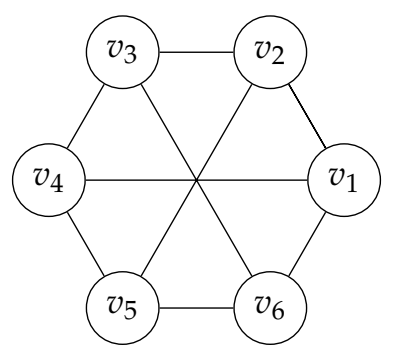

Figure 1. Graph $G$ which up to isomorphism can be exhausted by adding degree affinity edges say, $v_{2} v_{6}, v_{3} v_{5}$.

It follows from Figures 1 and 2 that applying the MDAC to $G$ and $H$ independently yields graphs $\mathbb{A}(G)$ with degree sequence $(4,4,4,4,3,3)$ and $\mathbb{A}(H)$ with degree sequence $(5,5,5,5,5,5)$. Hence, $\mathbb{A}(G)$ and $\mathbb{A}(H)$ remain disjoint in $\mathbb{A}(G) \cup \mathbb{A}(H)$. The second approach, to first add degree affinity edges $v_{i} u_{j}, 1 \leq i, j \leq 6$ 


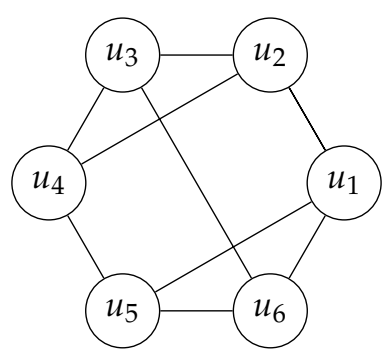

Figure 2. Graph $H$ reaches completeness in two iterations by adding the degree affinity edges say, (i) $u_{1} u_{4}, u_{2} u_{6}$, $u_{3} u_{5}$ then, (ii) $u_{1} u_{3}, u_{2} u_{5}, u_{4} u_{6}$.

(external to both $G$ and $H$ ) and then adding the degree affinity edges $v_{i} v_{j}$ and $u_{i} u_{j}, i \neq j, 1 \leq i, j \leq 6$ to $G^{+6}$ and $\mathrm{H}^{+6}$ yields the maximum number of degree affinity edges.

\subsection{Disjoint union of cycles}

Recall that a cycle on $n \geq 3$ vertices is a graph denoted by, $C_{n}$ and $V\left(C_{n}\right)=\left\{v_{i}: 1 \leq i \leq n\right\}$, $E\left(C_{n}\right)=\left\{v_{i} v_{i}+1: 1 \leq i \leq n-1\right\} \cup\left\{v_{n} v_{1}\right\}$. The family of 2-regular graphs are all graphs such that, each graph $G$ consists of one or more (disconnected or disjoint union of) cycles. The numbers $a_{n}$, $n=1,2,3,4,5,6,7,8,9,10, \ldots$ of 2-regular graphs on $n$ vertices are given by $0,0,1,1,1,2,2,3,4,5, \ldots, p(t)-$ $p(t-1)-p(t-2)+p(t-3), \ldots$ with $p(t)$ the partition function, (see https://oeis.org/A008483).

The results for both even and odd cycles are provided by Theorem 1 and Corollary 1. Categories of disconnected of 2-regular graphs (disjoint union of cycles) of order $3 \leq n \leq 10$ will be discussed.

Clearly, for $n=3,4,5$ there exists a unique 2-regular graph each i.e. $C_{3}, C_{4}, C_{5}$. The other categories are;

(i) $C_{3} \cup C_{3}$,

(ii) $C_{3} \cup C_{4}$,

(iii) $C_{3} \cup C_{5}$ and $C_{4} \cup C_{4}$,

(iv) $C_{3} \cup C_{6}, C_{4} \cup C_{5}$ and $C_{3} \cup C_{3} \cup C_{3}$ (or $3 C_{3}$ ),

(v) $C_{3} \cup C_{7}, C_{4} \cup C_{6}, C_{5} \cup C_{5}$ (or $2 C_{5}$ ) and $2 C_{3} \cup C_{4}$.

Theorem 3 read together with Theorem 1 leads to a proposition which requires no further proof.

Proposition 1. (a) For the disjoint union of two copies of an even cycle $C_{n}, n \geq 4$ the MDAC exhausts after $k=$ $2 n-3$ iterations, $\eta\left(C_{n} \cup C_{n}\right)_{n, \text { even }}=n(n-3)$ and $\mathbb{A}\left(C_{n} \cup C_{n}\right) \cong K_{2 n}$.

(b) For the disjoint union of two copies of an odd cycle $C_{n}, n \geq 3$ the MDAC exhausts after $k=2 n-3$ iterations, $\eta\left(C_{n} \cup C_{n}\right)_{n, o d d}=2 n^{2}-5 n+6$.

Definition 1. For a graph $G$ of order $n$ and $1 \leq t \leq n$, select $X \subseteq V(G),|X|=t$ such that a minimum number of vertices $v \in X$ are adjacent. The set $X$ is said to be an optimal near-independent selection.

For $X \subseteq V(G)$ the subgraph induced by $X$ is denoted by, $\langle X\rangle$. Definition 1 can be put differently i.e. select $X \subseteq V(G),|X|=t$ such that $\langle X\rangle$ has a minimum number of edges.

Proposition 2. $\quad$ (i) $\eta\left(C_{3} \cup C_{3}\right)=9$.

(ii) $\eta\left(C_{3} \cup C_{4}\right)=10$.

(iii) (a). $\eta\left(C_{3} \cup C_{5}\right)=11$.

(b). $\eta\left(C_{4} \cup C_{4}\right)=20$.

(iv) (a). $\eta\left(C_{3} \cup C_{6}\right)=11$.

(b). $\eta\left(C_{4} \cup C_{5}\right)=21$.

(c). $\eta\left(C_{3} \cup C_{3} \cup C_{3}\right) \geq 19$.

(v) (a). $\eta\left(C_{3} \cup C_{7}\right)=19$.

(b). $\eta\left(C_{4} \cup C_{6}\right)=24$. 
(c). $\eta\left(C_{5} \cup C_{5}\right)=31$.

(d). $\eta\left(2 C_{3} \cup C_{4}\right) \geq 21$.

Proof. (i) Follows from Proposition 1.

(ii) Let $C_{3}$ be on vertices $v_{1}, v_{2}, v_{3}$ and $C_{4}$ on vertices $u_{1}, u_{2}, u_{3}, u_{4}$. Without loss of generality add the external degree affinity edges between $v_{1}, v_{2}, v_{3}$ and $u_{1}, u_{2}, u_{3}$ in 3 iterations. Now vertices $u_{1}, u_{2}, u_{3}$ each, has degree of 5 . Add degree affinity edge $u_{1} u_{3}$ to exhaust the MDAC. Clearly, $\eta\left(C_{3} \cup C_{4}\right)=10$.

(iii) (a). Let $C_{3}$ be on vertices $v_{1}, v_{2}, v_{3}$ and $C_{5}$ on vertices $u_{1}, u_{2}, u_{3}, u_{4}, u_{5}$. Note that if degree affinity edges between $v_{1}, v_{2}, v_{3}$ and $u_{1}, u_{2}, u_{3}$ are added then $u_{4}, u_{5}$ remain adjacent. An optimal near-independent selection will, without loss of generality be say, vertices $u_{1}, u_{2}, u_{4}$. Therefore, in $1^{\text {st }}$-iteration add the degree affinity edges $u_{3} u_{5}, v_{1} u_{1}, v_{2} u_{2}, v_{3} u_{4}$. During the $2^{\text {nd }}$ - and $3^{\text {rd }}$-iteration reach completion between $v_{1}, v_{2}, v_{3}$ and $u_{1}, u_{2}, u_{4}$. In the exhaustive $4^{t h}$-iteration add either $u_{1} u_{4}$ or $u_{2} u_{4}$. Clearly, $\eta\left(C_{3} \cup C_{5}\right)=11$.

(b). Follows from Proposition 1.

(iv) (a). Let $C_{3}$ be on vertices $v_{1}, v_{2}, v_{3}$ and $C_{6}$ on vertices $u_{1}, u_{2}, u_{3}, u_{4}, u_{5}, u_{6}$. Without loss of generality an optimal near-independent selection of vertices in $C_{6}$ will be say, $u_{1}, u_{3}, u_{5}$. Hence, in three iterations add the degree affinity edges by first adding, either $u_{2} u_{4}$ or $u_{2} u_{6}$ or $u_{4} u_{6}$ together with $v_{1} u_{1}, v_{2} u_{2}$, $v_{3} u_{3}$. Thereafter complete the degree affinity edges between $C_{3}$ and $C_{6}$. Finally, add either $u_{1} u_{3}$ or $u_{1} u_{5}$ or $u_{3} u_{5}$. Clearly, $\eta\left(C_{3} \cup C_{6}\right)=11$.

(b). The result follows through similar reasoning in the proof of (ii).

(c). Let the vertices of three copies of $C_{3}$ be labeled $v_{1}, v_{2}, v_{3}$ and $u_{1}, u_{2}, u_{3}$ and $w_{1}, w_{2}, w_{3}$, respectively. For the convenience of reference label the cycles, $G_{1}, G_{2}, G_{3}$ respectively.

Case 1: Add all the degree affinity edges between any pair of $C_{3}$ cycles. Since the MDAC is exhausted, $\eta\left(C_{3} \cup C_{3} \cup C_{3}\right) \geq 9$.

Case 2: In the $1^{\text {st }}$-iteration consider pairs of cycles in the order, $\left(G_{1}, G_{2}\right),\left(G_{2}, G_{3}\right),\left(G_{3}, G_{1}\right),\left(G_{1}, G_{2}\right)$ and add the degree affinity edges say, $v_{1} u_{1}, u_{2} w_{2}, w_{3} v_{3}, v_{2} u_{3}$. In the $2^{\text {nd }}$-iteration consider pairs of cycles in the order, $\left(G_{2}, G_{3}\right),\left(G_{3}, G_{1}\right),\left(G_{1}, G_{2}\right)$ and add the degree affinity edges say, $u_{1} w_{2}, w_{3} v_{1}$, $v_{2} u_{2}, v_{3} u_{3}$. In the $3^{r d}$-iteration consider pairs of cycles in the order, $\left(G_{3}, G_{1}\right),\left(G_{1}, G_{2}\right)$ and add the degree affinity edges say, $w_{2} v_{3}, w_{3} u_{3}, v_{1} u_{2}, v_{2} u_{1}$. In the $4^{\text {th }}$-iteration consider pairs of cycles in the order, $\left(G_{1}, G_{2}\right),\left(G_{2}, G_{3}\right),\left(G_{3}, G_{1}\right)$ and add the degree affinity edges say, $v_{1} u_{3}, v_{3} u_{1}, u_{2} w_{3}, w_{2} v_{2}$. Finally to exhaust the MADC add degree affinity edges, $v_{2} w_{3}, v_{1} w_{2}, v_{3} u_{2}$. Since the methodology has only been tested exhaustively and not proven to yield the maximum number of degree affinity edges the best result is, $\eta\left(C_{3} \cup C_{3} \cup C_{3}\right) \geq \max \{9,19\}=19$.

(v) (a). Case 1: Clearly since $C_{3}$ is complete, $\eta\left(C_{3} \cup C_{7}\right) \geq 10=\eta\left(C_{7}\right)$.

Case 2: Let $C_{3}$ be on vertices $v_{1}, v_{2}, v_{3}$ and $C_{7}$ on vertices $u_{1}, u_{2}, u_{3}, u_{4}, u_{5}, u_{6}, u_{7}$. It follows easily that if the MDAC is applied between $C_{3}$ and vertices $u_{1}, u_{2}, u_{3}$ then, $\eta\left(C_{3} \cup C_{7}\right) \geq 13$.

Case 3: Apply the MDAC between $C_{3}$ and vertices $u_{1}, u_{2}, u_{4}$ as follows. Add degree affinity edges $v_{1} u_{1}, v_{2} u_{2}, v_{3} u_{4}$ as well as say, $u_{3} u_{6}, u_{5} u_{7}$. In the $2^{n d}$-iteration add, $v_{1} u_{2}, v_{2} u_{4}, v_{3} u_{1}$ as well as say, $u_{3} u_{5}$. In the $3^{r d}$-iteration add $v_{1} u_{4}, v_{2} u_{1}, v_{3} u_{2}$. Exhaust the MDAC in the $4^{\text {th }}$-iteration by adding say, $u_{1} u_{4}$. Hence, $\eta\left(C_{3} \cup C_{7}\right) \geq 13$.

Case 4: Apply the MDAC between $C_{3}$ and vertices $u_{1}, u_{2}, u_{5}$. Through similar reasoning as in Case 3 it follows that, $\eta\left(C_{3} \cup C_{7}\right) \geq 14$.

Case 5: Without loss of generality an optimal near-independent selection of vertices in $C_{7}$ will be say, $u_{1}, u_{3}, u_{5}$. In the $1^{s t}$-iteration add the degree affinity edges, $v_{1} u_{1}, v_{2} u_{3}, v_{3} u_{5}$ and $u_{2} u_{7}, u_{4} u_{6}$. In the $2^{\text {nd }}$-iteration ad the degree affinity edges $v_{1} u_{3}, v_{3} u_{1}, v_{2} u_{5}, u_{2} u_{6}, u_{4} u_{7}$. In the $3^{\text {rd }}$-iteration ad the degree affinity edges $v_{1} u_{5}, v_{2} u_{1}, v_{3} u_{3}, u_{2} u_{4}$. In the $3^{r d}$-iteration ad the degree affinity edges $v_{1} u_{2}, v_{3} u_{4}, u_{1} u_{5}$. Finally, add $v_{1} u_{4}$ and $u_{2} u_{5}$. Since all possibilities up to isomorphisms have been considered the result is, $\eta\left(C_{3} \cup C_{7}\right)=\max \{10,13$ (repeated), 14,19$\}=19$.

(b) Let $C_{4}$ be on vertices $v_{1}, v_{2}, v_{3}, v_{4}$ and $C_{6}$ on vertices $u_{1}, u_{2}, u_{3}, u_{4}, u_{5}, u_{6}$.

Case 1: If the MDAC is applied to $C_{4}$ and $C_{6}$ independently it follows that $\eta\left(C_{4} \cup C_{6}\right) \geq 11$. 
Case 2: Let $C_{4}$ be on vertices $v_{1}, v_{2}, v_{3}, v_{4}$ and $C_{6}$ on vertices $u_{1}, u_{2}, u_{3}, u_{4}, u_{5}, u_{6}$. By applying the MDAC between $C_{4}$ and vertices $u_{1}, u_{2}, u_{3}, u_{4}$ it follows easily that exhaustion is reached between six iterations. Hence, $\eta\left(C_{4} \cup C_{6}\right) \geq 21$.

Case 3: By applying the MDAC between $C_{4}$ and vertices $u_{1}, u_{2}, u_{3}, u_{5}$ it follows easily that exhaustion is reached between six iterations. Note that in the $1^{s t}$-iteration the degree affinity edge $u_{4} u_{6}$ was added thus, $\eta\left(C_{4} \cup C_{6}\right) \geq 22$.

Case 4: By applying the MDAC between $C_{4}$ and vertices $u_{1}, u_{2}, u_{4}, u_{5}$ it follows easily that exhaustion is reached between six iterations. Note that in the $1^{\text {st }}$-iteration the degree affinity edge $u_{3} u_{6}$ was added thus, $\eta\left(C_{4} \cup C_{6}\right) \geq 23$. Since all possibilities up to isomorphisms have been considered the result is, $\eta\left(C_{4} \cup C_{6}\right)=\max \{11,21,22,23\}=24$.

(c). Follows from Proposition 1.

(d). Through similar reasoning as that in (iv)(c) the result is, $\eta\left(2 C_{3} \cup C_{4}\right) \geq 21$.

Another approach to find the results (iv)(c) and (v)(a)-(d) is proposed. Consider (iv)(c). In the first iteration add the degree affinity edges say, $v_{1} u_{1}, u_{2} w_{2}, w_{3} v_{3}$ and $v_{2} u_{3}$. Relabel the vertices as follows: $v_{1} \mid z_{1}$, $u_{1}\left|z_{2}, u_{3}\right| z_{3}, u_{2}\left|z_{4}, w_{2}\right| z_{5}, w_{1}\left|z_{6}, w_{3}\right| z_{7}, v_{3}\left|z_{8}, v_{2}\right| z_{9}$. A chorded cycle as depicted in Figure 3 is obtained.

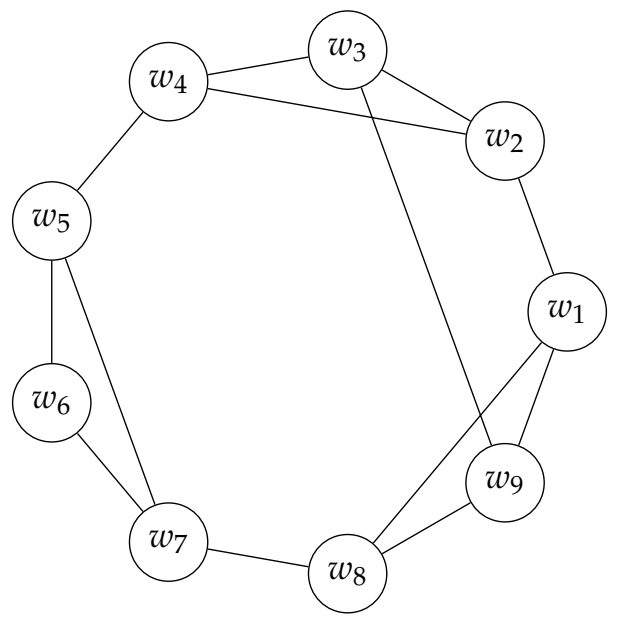

Figure 3. Chorded cycle.

The avenue for researching chorded cycles could lead to an improved methodology.

\subsection{Chorded cycles}

A chorded cycle with $\ell$ chords is denoted by $C_{n}^{\sim \ell}, 1 \leq \ell \leq \frac{n(n-3)}{2}$. The observations from Figures 1 and 2 read together with Theorem 1 and Corollary 1 provide a proposition which requires no further proof.

Proposition 3. For a cycle $C_{n}$,

(a) $\eta\left(C_{n}^{\sim \ell}\right) \leq \frac{n(n-3)}{2}-\ell$ if $n$ is even.

(b) $\eta\left(C_{n}^{\sim \ell}\right) \leq \frac{(n-2)(n-3)}{2}-\ell$ if $n$ is odd.

Chorded cycles with $\ell$ independent chords, i.e., no pair of distinct chords share an end-vertex will be denoted by $C_{n}^{\sim \ell(i)}, 1 \leq \ell \leq\left\lfloor\frac{n}{2}\right\rfloor$. A researcher, Dillon Lareau investigated a problem which is described as, finding "the number of ways of dividing $n$ labeled items into $k$ unlabeled boxes as evenly as possible". In the graph coloring context the Lareau problem can be stated as, finding "the number of ways of coloring $n$ labeled and isolated vertices (or the labeled vertices of the null graph, $\mathfrak{N}_{n}$ ) with $k$ distinct colors as evenly as possible". The aforesaid problem was investigated in the context of chromatic completion of graphs. For an introduction to chromatic completion of a graph $G$, see [5-9]. In the aforesaid context the number of ways of coloring was called the lucky number denoted by, $L(n, k)$. The vertex set partitions which correspond to the 
"ways of coloring" are called lucky partitions. A closed formula was announce by Dillon Lareau (11 June 2019) which is given by, $L(n, k)=\frac{n !}{A ! B !\left(\left[\frac{n}{k} ! !\right)^{A}\left(\left\lfloor\frac{n}{k} ! !\right)^{B}\right.\right.}, A=n(\bmod k), B=k-A$, (see https://oeis.org/A308624). Let the number of ways $\ell$ independent chords can be added to $C_{n}$ be denoted by, $\oplus_{\ell}\left(C_{n}\right)$.

Determining $\bigoplus_{\ell}\left(C_{n}\right)$ presents difficulty because no computer algorithm is available to generate the required partitions. To illustrate this difficulty consider finding all 2-chorded cycles of order 6 . To begin, first find the corresponding $L(6,(6-2))=\frac{6 !}{2 ! 2 !(2 !)^{2}(1 !)^{2}}=45$ lucky partitions.

The corresponding lucky partitions of $V\left(\mathfrak{N}_{6}\right)$ (null graph order 6 ) are;

$$
\begin{aligned}
& \left\{\left\{v_{1}, v_{2}\right\},\left\{v_{3}, v_{4}\right\},\left\{v_{5}\right\},\left\{v_{6}\right\}\right\}, \\
& \left\{\left\{v_{1}, v_{2}\right\},\left\{v_{3}, v_{5}\right\},\left\{v_{4}\right\},\left\{v_{6}\right\}\right\}, \\
& \left\{\left\{v_{1}, v_{2}\right\},\left\{v_{3}\right\},\left\{v_{4}, v_{5}\right\},\left\{v_{6}\right\}\right\}, \\
& \left\{\left\{v_{1}, v_{2}\right\},\left\{v_{3}, v_{6}\right\},\left\{v_{4}\right\},\left\{v_{5}\right\}\right\}, \\
& \left\{\left\{v_{1}, v_{2}\right\},\left\{v_{3}\right\},\left\{v_{4}, v_{6}\right\},\left\{v_{5}\right\}\right\}, \\
& \left\{\left\{v_{1}, v_{2}\right\},\left\{v_{3}\right\},\left\{v_{4}\right\},\left\{v_{5}, v_{6}\right\}\right\}, \\
& \left\{\left\{v_{1}, v_{3}\right\},\left\{v_{2}, v_{4}\right\},\left\{v_{5}\right\},\left\{v_{6}\right\}\right\}, \\
& \left\{\left\{v_{1}, v_{3}\right\},\left\{v_{2}, v_{5}\right\},\left\{v_{4}\right\},\left\{v_{6}\right\}\right\}, \\
& \left\{\left\{v_{1}, v_{3}\right\},\left\{v_{2}\right\},\left\{v_{4}, v_{5}\right\},\left\{v_{6}\right\}\right\}, \\
& \left\{\left\{v_{1}, v_{3}\right\},\left\{v_{2}, v_{6}\right\},\left\{v_{4}\right\},\left\{v_{5}\right\}\right\}, \\
& \left\{\left\{v_{1}, v_{3}\right\},\left\{v_{2}\right\},\left\{v_{4}, v_{6}\right\},\left\{v_{5}\right\}\right\}, \\
& \left\{\left\{v_{1}, v_{3}\right\},\left\{v_{2}\right\},\left\{v_{4}\right\},\left\{v_{5}, v_{6}\right\}\right\}, \\
& \left\{\left\{v_{1}, v_{4}\right\},\left\{v_{2}, v_{3}\right\},\left\{v_{5}\right\},\left\{v_{6}\right\}\right\}, \\
& \left\{\left\{v_{1}, v_{5}\right\},\left\{v_{2}, v_{3}\right\},\left\{v_{4}\right\},\left\{v_{6}\right\}\right\}, \\
& \left\{\left\{v_{1}\right\},\left\{v_{2}, v_{3}\right\},\left\{v_{4}, v_{5}\right\},\left\{v_{6}\right\}\right\}, \\
& \left\{\left\{v_{1}, v_{6}\right\},\left\{v_{2}, v_{3}\right\},\left\{v_{4}\right\},\left\{v_{5}\right\}\right\}, \\
& \left\{\left\{v_{1}\right\},\left\{v_{2}, v_{3}\right\},\left\{v_{4}, v_{6}\right\},\left\{v_{5}\right\}\right\}, \\
& \left\{\left\{v_{1}\right\},\left\{v_{2}, v_{3}\right\},\left\{v_{4}\right\},\left\{v_{5}, v_{6}\right\}\right\}, \\
& \left\{\left\{v_{1}, v_{4}\right\},\left\{v_{2}, v_{5}\right\},\left\{v_{3}\right\},\left\{v_{6}\right\}\right\}, \\
& \left\{\left\{v_{1}, v_{4}\right\},\left\{v_{2}\right\},\left\{v_{3}, v_{5}\right\},\left\{v_{6}\right\}\right\}, \\
& \left\{\left\{v_{1}, v_{4}\right\},\left\{v_{2}, v_{6}\right\},\left\{v_{3}\right\},\left\{v_{5}\right\}\right\}, \\
& \left\{\left\{v_{1}, v_{4}\right\},\left\{v_{2}\right\},\left\{v_{3}, v_{6}\right\},\left\{v_{5}\right\}\right\}, \\
& \left\{\left\{v_{1}, v_{4}\right\},\left\{v_{2}\right\},\left\{v_{3}\right\},\left\{v_{5}, v_{6}\right\}\right\}, \\
& \left\{\left\{v_{1}, v_{5}\right\},\left\{v_{2}, v_{4}\right\},\left\{v_{3}\right\},\left\{v_{6}\right\}\right\}, \\
& \left\{\left\{v_{1}\right\},\left\{v_{2}, v_{4}\right\},\left\{v_{3}, v_{5}\right\},\left\{v_{6}\right\}\right\}, \\
& \left\{\left\{v_{1}, v_{6}\right\},\left\{v_{2}, v_{4}\right\},\left\{v_{3}\right\},\left\{v_{5}\right\}\right\}, \\
& \left\{\left\{v_{1}\right\},\left\{v_{2}, v_{4}\right\},\left\{v_{3}, v_{6}\right\},\left\{v_{5}\right\}\right\}, \\
& \left\{\left\{v_{1}\right\},\left\{v_{2}, v_{4}\right\},\left\{v_{3}\right\},\left\{v_{5}, v_{6}\right\}\right\}, \\
& \left\{\left\{v_{1}, v_{5}\right\},\left\{v_{2}\right\},\left\{v_{3}, v_{4}\right\},\left\{v_{6}\right\}\right\}, \\
& \left\{\left\{v_{1}\right\},\left\{v_{2}, v_{5}\right\},\left\{v_{3}, v_{4}\right\},\left\{v_{6}\right\}\right\}, \\
& \left\{\left\{v_{1}, v_{6}\right\},\left\{v_{2}\right\},\left\{v_{3}, v_{4}\right\},\left\{v_{5}\right\}\right\}, \\
& \left\{\left\{v_{1}\right\},\left\{v_{2}, v_{6}\right\},\left\{v_{3}, v_{4}\right\},\left\{v_{5}\right\}\right\}, \\
& \left\{\left\{v_{1}\right\},\left\{v_{2}\right\},\left\{v_{3}, v_{4}\right\},\left\{v_{5}, v_{6}\right\}\right\}, \\
& \left\{\left\{v_{1}, v_{5}\right\},\left\{v_{2}, v_{6}\right\},\left\{v_{3}\right\},\left\{v_{4}\right\}\right\}, \\
& \left\{\left\{v_{1}, v_{5}\right\},\left\{v_{2}\right\},\left\{v_{3}, v_{6}\right\},\left\{v_{4}\right\}\right\}, \\
& \left\{\left\{v_{1}, v_{5}\right\},\left\{v_{2}\right\},\left\{v_{3}\right\},\left\{v_{4}, v_{6}\right\}\right\}, \\
& \left\{\left\{v_{1}, v_{6}\right\},\left\{v_{2}, v_{5}\right\},\left\{v_{3}\right\},\left\{v_{4}\right\}\right\}, \\
& \left\{\left\{v_{1}\right\},\left\{v_{2}, v_{5}\right\},\left\{v_{3}, v_{6}\right\},\left\{v_{4}\right\}\right\}, \\
& \left\{\left\{v_{1}\right\},\left\{v_{2}, v_{5}\right\},\left\{v_{3}\right\},\left\{v_{4}, v_{6}\right\}\right\}, \\
& \left\{\left\{v_{1}, v_{6}\right\},\left\{v_{2}\right\},\left\{v_{3}, v_{5}\right\},\left\{v_{4}\right\}\right\}, \\
& \left\{\left\{v_{1}\right\},\left\{v_{2}, v_{6}\right\},\left\{v_{3}, v_{5}\right\},\left\{v_{4}\right\}\right\}, \\
& \left\{\left\{v_{1}\right\},\left\{v_{2}\right\},\left\{v_{3}, v_{5}\right\},\left\{v_{4}, v_{6}\right\}\right\}, \\
& \left\{\left\{v_{1}, v_{6}\right\},\left\{v_{2}\right\},\left\{v_{3}\right\},\left\{v_{4}, v_{5}\right\}\right\}, \\
& \left\{\left\{v_{1}\right\},\left\{v_{2}, v_{6}\right\},\left\{v_{3}\right\},\left\{v_{4}, v_{5}\right\}\right\}, \\
& \left\{\left\{v_{1}\right\},\left\{v_{2}\right\},\left\{v_{3}, v_{6}\right\},\left\{v_{4}, v_{5}\right\}\right\} \text {. }
\end{aligned}
$$


Finally, eliminate all lucky partitions which have some 2-element subset which is an edge of the cycle $C_{6}$. This yields,

$$
\begin{aligned}
& \left\{\left\{v_{1}, v_{3}\right\},\left\{v_{2}, v_{4}\right\},\left\{v_{5}\right\},\left\{v_{6}\right\}\right\}, \\
& \left\{\left\{v_{1}, v_{3}\right\},\left\{v_{2}, v_{5}\right\},\left\{v_{4}\right\},\left\{v_{6}\right\}\right\}, \\
& \left\{\left\{v_{1}, v_{3}\right\},\left\{v_{2}, v_{6}\right\},\left\{v_{4}\right\},\left\{v_{5}\right\}\right\}, \\
& \left\{\left\{v_{1}, v_{3}\right\},\left\{v_{2}\right\},\left\{v_{4}, v_{6}\right\},\left\{v_{5}\right\}\right\}, \\
& \left\{\left\{v_{1}, v_{4}\right\},\left\{v_{2}, v_{5}\right\},\left\{v_{3}\right\},\left\{v_{6}\right\}\right\}, \\
& \left\{\left\{v_{1}, v_{4}\right\},\left\{v_{2}\right\},\left\{v_{3}, v_{5}\right\},\left\{v_{6}\right\}\right\}, \\
& \left\{\left\{v_{1}, v_{4}\right\},\left\{v_{2}, v_{6}\right\},\left\{v_{3}\right\},\left\{v_{5}\right\}\right\}, \\
& \left\{\left\{v_{1}, v_{4}\right\},\left\{v_{2}\right\},\left\{v_{3}, v_{6}\right\},\left\{v_{5}\right\}\right\}, \\
& \left\{\left\{v_{1}, v_{5}\right\},\left\{v_{2}, v_{4}\right\},\left\{v_{3}\right\},\left\{v_{6}\right\}\right\}, \\
& \left\{\left\{v_{1}\right\},\left\{v_{2}, v_{4}\right\},\left\{v_{3}, v_{5}\right\},\left\{v_{6}\right\}\right\}, \\
& \left\{\left\{v_{1}\right\},\left\{v_{2}, v_{4}\right\},\left\{v_{3}, v_{6}\right\},\left\{v_{5}\right\}\right\}, \\
& \left\{\left\{v_{1}, v_{5}\right\},\left\{v_{2}, v_{6}\right\},\left\{v_{3}\right\},\left\{v_{4}\right\}\right\}, \\
& \left\{\left\{v_{1}, v_{5}\right\},\left\{v_{2}\right\},\left\{v_{3}, v_{6}\right\},\left\{v_{4}\right\}\right\}, \\
& \left\{\left\{v_{1}, v_{5}\right\},\left\{v_{2}\right\},\left\{v_{3}\right\},\left\{v_{4}, v_{6}\right\}\right\}, \\
& \left\{\left\{v_{1}\right\},\left\{v_{2}, v_{5}\right\},\left\{v_{3}, v_{6}\right\},\left\{v_{4}\right\}\right\}, \\
& \left\{\left\{v_{1}\right\},\left\{v_{2}, v_{5}\right\},\left\{v_{3}\right\},\left\{v_{4}, v_{6}\right\}\right\}, \\
& \left\{\left\{v_{1}\right\},\left\{v_{2}, v_{6}\right\},\left\{v_{3}, v_{5}\right\},\left\{v_{4}\right\}\right\}, \\
& \left\{\left\{v_{1}\right\},\left\{v_{2}\right\},\left\{v_{3}, v_{5}\right\},\left\{v_{4}, v_{6}\right\}\right\} .
\end{aligned}
$$

Clearly, $\oplus_{2}\left(C_{6}\right)=18$

Corollary 2. For a cycle $C_{n}, n \geq 3$, we have the inequality, $\bigoplus_{\ell}\left(C_{n}\right)<L(n, n-\ell)$.

Proof. Since some lucky partitions have at least one 2-element subset which is an edge of the cycle $C_{n}$, the result holds.

Let a family of non-isomorphic independent $\ell$-chorded cycles be denoted by, $\mathfrak{C}_{n}^{\sim \ell(i)}$. For the example above and without loss of generality it follows that,

$$
\mathfrak{C}_{6}^{\sim 2(i)}=\left\{\left\{\left\{v_{1}, v_{3}\right\},\left\{v_{2}, v_{4}\right\},\left\{v_{5}\right\},\left\{v_{6}\right\}\right\},\left\{\left\{v_{1}, v_{3}\right\},\left\{v_{2}, v_{5}\right\},\left\{v_{4}\right\},\left\{v_{6}\right\}\right\},\left\{\left\{v_{1}, v_{3}\right\},\left\{v_{2}\right\},\left\{v_{4}, v_{6}\right\},\left\{v_{5}\right\}\right\},\right.
$$
$\left.\left\{\left\{v_{1}, v_{4}\right\},\left\{v_{2}, v_{5}\right\},\left\{v_{3}\right\},\left\{v_{6}\right\}\right\}\right\}$.

We now open the avenue for researching the degree affinity properties of $C_{n} \cup C_{m}, m>n \geq 3$. If all external degree affinity edges between $C_{n}$ and $C_{m}$ as well as the required internal degree affinity edges to $C_{m}$ is added during the $1^{\text {st }}$-iteration of the MDAC, a Hamiltonian graph is obtained. This Hamiltonian graph is a chorded cycle, $C_{n+m}^{\sim \ell(i)}, \ell=n+\left\lfloor\frac{m-n}{2}\right\rfloor \geq 3$. The Hamilton cycle is not unique because the choice of connecting pairs of vertices between $C_{n}$ and $C_{m}$ is not unique. By exhausting the ways in which the pairs of vertices can be connected and by exhausting the Hamilton cycles within each chorded cycle, a family $\mathfrak{C}_{n+m}^{\sim \ell(i)}$ can be generated. Therefore, from Definition 2.1 in [4] it follows that, for $m \geq n, \eta\left(C_{n} \cup C_{m}\right) \leq\left(n+\left\lfloor\frac{m-n}{2}\right\rfloor\right)+\max \left\{\eta\left(C_{n+m}^{\sim \ell(i)}\right)\right.$ : $\left.C_{n+m}^{\sim \ell(i)} \in \mathfrak{C}_{n+m}^{\sim \ell(i)}, \ell=n+\left\lfloor\frac{m-n}{2}\right\rfloor\right\}$.

Example 1. Consider $C_{3} \cup C_{3}$. Let the cycles be on vertices $v_{1}, v_{2}, v_{3}$ and $u_{1} u_{2}, u_{3}$ respectively. Without loss of generality add the three external degree affinity edges, $v_{1} u_{1}, v_{2} u_{2}, v_{3} u_{3}$. Relabel the vertices as follows: $v_{1} \mid z_{1}$, $v_{3}\left|z_{2}, v_{2}\right| z_{3}, u_{2}\left|z_{4}, u_{3}\right| z_{5}, u_{1} \mid z_{6}$.

It implies that $\eta\left(C_{3} \cup C_{3}\right) \leq 3+\max \{2,6\}=9$. Note that since the $C_{3}^{\prime}$ are complete, a unique independent chorded cycle $C_{6}^{\sim 3(i)}$ is obtained which yields equality. Hence, $\eta\left(C_{3} \cup C_{3}\right)=9$.

\section{Conclusion}

Besides doing "mathematics for the sake of mathematics", motivation related to applications of the notion of degree affinity has been stated in [4]. Current research into an application related to chemical affinity between atoms or molecular affinity in molecular structures is underway. It is hoped to report on results soon. 

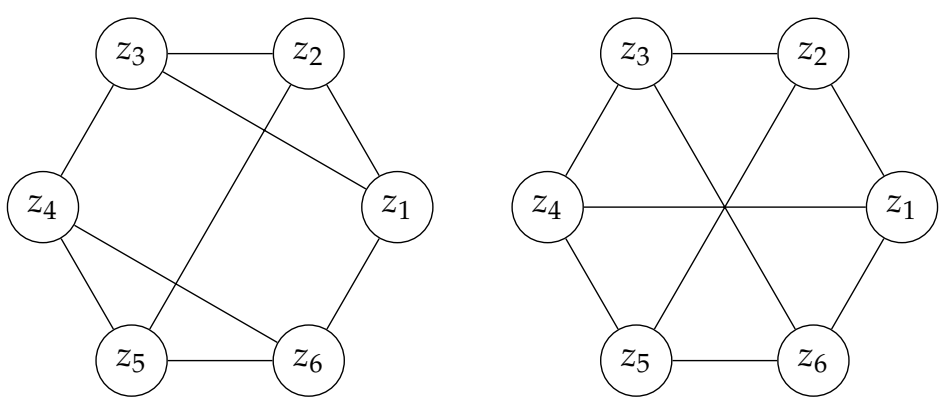

Figure 4. $\mathfrak{C}_{6}^{\sim 3(i)}$ has two distinct $C_{6}^{\sim 3(i)}$.

Investigating the new parameter $\eta(G)$ for the disjoint union of cycles poses numerous challenges. It is the considered view of the author that for labeled graphs, the development of a computer generator of lucky partitions followed by the reduction of the partitions to the permissible partitions is key to furthering this research meaningfully. If a methodology can be developed to generate a family of non-isomorphic $\ell$-chorded cycles it will be worthy to further results.

Finding a closed formula for $\bigoplus_{\ell}\left(C_{n}\right), n \geq 3$ is worthy of endeavour. Finding improvement on the inequality of Corollary 2 remains open.

Acknowledgments: The author would like to thank the anonymous referees for their constructive comments, which helped to improve on the elegance of this paper.

Conflicts of Interest: “The author declares no conflict of interest."

\section{References}

[1] Bondy, J. A., \& Murty, U. S. R. (1976). Graph Theory with Applications. Macmillan Press, London.

[2] Harary, F. (1969). Graph Theory. Addison-Wesley, Reading MA.

[3] West, B. (1996). Introduction to Graph Theory. Prentice-Hall, Upper Saddle River.

[4] Kok, J. Degree affinity number of graphs, Communicated.

[5] Kok, J. (2020). Chromatic completion number of corona of path and cycle graphs. Malaya Journal of Matematik, 8(1), $1-6$.

[6] Kok, J. (2020). Lucky $\chi$-polynomial of graphs of order 5. Malaya Journal of Matematik, 8(3), 767-774.

[7] Kok, J. (2020). A note on perfect lucky k-colourable graphs. Journal of Mathematics and Computer Science, 21, 192-197.

[8] Kok, J. (2019). Heuristic method to determine lucky $k$-polynomials for $k$-colorable graphs. Acta Universitatis Sapientiae, Informatica, 11(2), 205-2013.

[9] Kok, J. (2020). Errata: Heuristic method to determine lucky $k$-polynomials for $k$-colorable graphs. Acta Universitatis Sapientiae, Informatica, 12(1), 158-158. 